\title{
Research Article Study on Optimization of Night Illumination in Expressway Long Tunnels
}

\author{
Weihua Zhao, Hao Chen, Qiang Yu, and Haoxue Liu \\ School of Automobile, Chang'an University, Xi'an 710064, China \\ Correspondence should be addressed to Weihua Zhao, sdzwh@chd.edu.cn
}

Received 11 September 2012; Revised 6 November 2012; Accepted 21 November 2012

Academic Editor: Wuhong Wang

Copyright (c) 2012 Weihua Zhao et al. This is an open access article distributed under the Creative Commons Attribution License, which permits unrestricted use, distribution, and reproduction in any medium, provided the original work is properly cited.

Night illumination of expressway long tunnels in China was far from drivers' demand and caused lots of traffic accidents and electricity wasting. In order to solve this problem, reasonable night luminosity in expressway long tunnels in China was studied. Based on a visual disturbance generation mechanism, the mistakes in night illumination of Chinese expressway long tunnels were analyzed. Real road experiments were conducted and fuzzy assessments were used to establish models. The results showed that night luminosity was too high and should be controlled below $9 \mathrm{Lx}$. In the entrance segment, the luminosity should be gradually increased from $0 \mathrm{Lx}$ to $9 \mathrm{Lx}$. In the middle segment, the luminosity should be remained at $9 \mathrm{Lx}$. In the exit segment, the luminosity should be gradually decreased to $0 \mathrm{Lx}$. The optimized luminosity is highly consistent with the actual demand of drivers. Traffic safety was improved and electricity consumption was reduced.

\section{Introduction}

The environment inside and outside tunnels was of great difference, in particular the luminosity. Significant contrast of dark and bright increased the visual and psychological burden of drivers. In the daytime, the drivers would suffer dark adaptation when entering tunnels and light adaptation when leaving tunnels. While at night, drivers would suffer light adaptation when entering these tunnels and dark adaptation when leaving. All expressway long tunnels in China are equipped with lights. The ventilation and lighting specification of PRC only required luminosity in day [1]. The same illumination program were generally adopted in Chinese practices for both day and night. This illumination method worsen visual disturbance in night and increased electricity consumption. For example the expense of illumination electricity in Chinese Zhongnan mountain tunnel was higher than charge. The night luminosity in expressway long tunnel was too higher to meet the drivers' demand. 


\section{Literature Review}

The Luminosity difference inside tunnel and outside was a major factor to traffic safety. At the same time, illumination consumption of large amounts of electricity was obvious. Many studies have been conducted about this problem.

The ventilation and lighting specification of PRC stipulated detailed parameters of luminosity of tunnels in daytime [1]. The specification ignored the fact that drivers would suffer different visual disturbances between day and night. It stipulated luminosity mainly based on the visual disturbance that drivers would suffer in tunnel in daytime. Environmental factors affected driving behavior and information perception [2,3]. Although the exact luminosity maybe different in every country, the major factors considered were the same. The different country had built self-specification $[1,4]$. International Commission on Illumination published "Guide for the Lighting of Road Tunnels and Underpasses" in 2004 [5]. Schreuder and Swart studied the energy saving in tunnel entrance lighting in order to decrease the electricity consumption [6]. Piarc also studied the problem of operational cost reduction of road tunnels and proposed the illumination solution [7]. In China, Xia analyzed some problems about tunnel illumination unsolved in the specification. He proposed that the problem should be studied from driver light and dark adaption but no luminosity value regarded as appropriate [8]. Qiu et al. studied the specification and compared it with CIE then proposed the dynamic requiring illumination in the transition section of the tunnel lighting [9]. Tu and Chen Studied the illumination parameter of short tunnels [10]. Zhang aims at medium and short tunnels in expressway studied illumination [11]. Tunnel entrance and exit were regarded as black points of traffic accident so, Wang et al., analyze the illumination design at tunnel entrance and exit [12]. Schreuder researched the illumination in tunnel entrances at day [13]. Zhao and Liu from Chang'an University, investigated drivers' visual feature variation pattern and driving behaviors in tunnels and established relevant models to describe the variation pattern [14]. Fukuda selected one tunnel to study the eye fixation point [15]. Wang et al. collected data of Chinese traffic accidents happened in tunnel and proposed the high rate segment in tunnel [16]. Du et al. from Tongi University evaluated driving safety level in tunnels using drivers' pupil area variation and put forward many conclusions. From the point of content and methods, this study attributed pupil area variation completely to drivers' nervousness and ignored the impact of illumination and dark adaptation time. When driving in tunnel the relation between visual information perception and safety was analyzed $[17,18]$. Schreuder, describes the relation between visual performance and road safety [19]. In china tunnel operation difficulties was researched including illumination [20]. In recent two years researchers in China mainly concerned in LED applied in tunnel [21]. All these studies were connected to the illumination in expressway tunnel but the conclusions could not be applied directly to the Chinese expressway long tunnel.

Although some studies on tunnel lighting were conducted, Chinese expressway long tunnel illumination at night is unreasonable yet. The reason for the above situation was that the illumination design ignored the sequence difference of dark and light adaptation between day and night, did not evaluate environment consistency, and only considered that illumination in tunnels could improve visual field. In contrast, the calculation method of illumination abroad was of great difference from the demand of drivers in China due to differences of vehicle velocity, traffic condition, and human factors. 


\section{Research Method and Experiment}

\subsection{Research Method}

It was difficult to find out the exact luminosity. The reason was that human factors were not easy to quantitatively describe. Whether the luminosity was satisfying to drivers' demand only depended on the divers assessment. But the luminosity that could be applied in experiments was restrict to real tunnel illumination. If this problem was researched in laboratory, the drivers could not obtain the same feeling from the stimulated environment compared with real driving environment.

To solve this significant problem, this paper would conduct the real rod experiments. Drivers passed through the tunnels with a different luminosity and assessed whether the illumination was appropriate. Considering the fact that people could not give exact assessment results fuzzy mathematics would be the best method to calculate the luminosity. Aiming at the calculated luminosity, some drivers were selected to verify whether the optimized luminosity was right. Via the above methods, the best illumination parameters would be certain. Based on these analyses, this paper researched appropriate night luminosity in expressway long tunnels fit for Chinese real character of traffic condition and human factors and proposed an optimized night lighting solution.

\subsection{Experiments}

\subsubsection{Subjects}

As the uniform, requirements for more than 2 years of expressway driving experience were required. Also, the drivers should have good driving skills and normal visual ability, without physiological defect, or serious or severe accident experience. The subjects were from different industries with different experiences. They had different occupations and years of driving. At the same time, age and gender were considered. At last, including 12 female drivers total, 37 drivers were selected in the experiments.

\subsubsection{Instruments}

Luminosity meter was used to measure luminosity on ground and wall in tunnels. A 5-seatcar was chosen as an experiment vehicle. The vehicle velocity variation was recorded by Microwave radar speed sensor.

\subsubsection{Tunnels and Luminosity}

Before they entered each experimental tunnel, the drivers were required to run on an expressway without light for more than 30 minutes to make sure of full dark adaptation to the luminosity outside the tunnels. All the tunnels were one-way traffic and without interference from vehicles in the other way. Other vehicles would not pose any impact on the ambient luminosity. The length and luminosity of each tunnel were shown in Table 1.

All the tunnels had the same night and day illumination. The luminosity in middle segment was lower than both ends. The illumination was enhanced in entrance and exit segment. The luminosity variation pattern could be seen in Figure 1. 
Table 1: Tunnels length and luminosity in middle segment.

\begin{tabular}{lccccc}
\hline Name of tunnel & Yongxing & Lijiahe & Nanwutai & Zhongnanshan & Qingcha \\
\hline Length/m & 1140 & 3650 & 2561 & 18020 & 1343 \\
Illumination/Lx & 60 & 50 & 40 & 30 & 20 \\
\hline
\end{tabular}

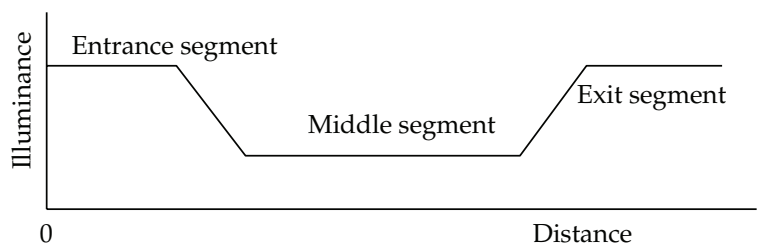

Figure 1: Luminosity variation pattern.

Table 2: Membership grade between luminosity and fuzzy assessment.

\begin{tabular}{lccccc}
\hline Luminosity & 20 & 30 & 40 & 50 & 60 \\
\hline Appropriate & 0.20 & 0.10 & 0.00 & 0.00 & 0.00 \\
Less appropriate & 1.00 & 0.31 & 0.00 & 0.00 & 0.00 \\
All right & 0.64 & 1.00 & 0.37 & 0.25 & 0.20 \\
Brighter & 0.12 & 0.73 & 1.00 & 0.90 & 0.85 \\
Too bight & 0.00 & 0.00 & 0.90 & 0.95 & 1.00 \\
\hline
\end{tabular}

\subsection{Experiment Program}

The drivers did not know the goal of experiment and other factors were controlled as much as possible. The drivers entered the tunnels with their own driving habits after they had full adaptation outside the tunnels on the expressway. The drivers made subjective assessment of the appropriateness of luminosity in course of driving. Assessment results were recorded by the examiner.

\section{Calculation and Analysis}

\subsection{Luminosity in Middle Segment}

To ease dark and light adaptation of drivers, transition of tunnel luminosity should be smooth and comfortable. The luminosity in entrance segment should be gradually increased to the luminosity value in the middle segment, then retained at an appropriate value in middle segment, and then gradually decreased to $0 \mathrm{Lx}$ in the exit segment. Therefore, the appropriate luminosity value in middle segment of expressway long tunnel was a critical parameter for illumination design.

\subsubsection{Fuzzy Assessment}

According to the illumination of the experimental tunnels, the domain of luminosity value in middle segment was taken as $L_{m}=\{60,50,40,30,20\}$. To simplify the assessment model, only the luminosity value in middle segment was assumed to be an assessment factor. Cognitive 
fuzzy set of luminosity value in middle segment of expressway long tunnel at night was established. Fuzzy set of luminosity appropriateness in middle segment: $D=\{$ too bright, lighter, all right, less appropriate, appropriate $\}$ and recorded as $\left\{D_{1}, D_{2}, D_{3}, D_{4}, D_{5}\right\}$. The membership grade of each was obtained using a fuzzy statistical method and was recorded as $R_{m}$. By taking all the results into consideration, the fuzzy relation between the luminosity value and appropriateness was obtained, as shown in Table 2.

Effective luminosity data of expressway long tunnel at night was obtained through luminosity assessment experiment. Using summary of assessment of tunnel luminosity cognition, membership grade of each fuzzy set of luminosity appropriateness was obtained. Membership grade variation showed that with the gradual decrease of luminosity value, the luminosity in middle segment was closer to the actual demand of drivers. This showed that the luminosity at night in middle segment of the Chinese expressway tunnels is too high.

\subsubsection{Model and Results}

Table 2 showed that the luminosity value in middle segment was smaller the membership grade of subset \{appropriate\} against the fuzzy set was higher. Considering road ambient consistency the luminosity in middle segment was smaller the ambient consistency, was higher. According to Weber's Law, the higher the illumination was, the smaller, equivalent variation of physical quantity that drivers can feel. On the basis of the above analysis and in combination of fuzzy mathematical membership function that fuzzy distribution of appropriate night luminosity in expressway long tunnels was "small" was certain. So that normal distribution was fit for the luminosity optimization function model. The specific calculation was shown in the following formula:

$$
\underset{\sim}{A}(x)= \begin{cases}1, & x \leq a \\ e^{-((x-a) / \sigma)^{2}}, & x>a\end{cases}
$$

where $\underset{\sim}{A}$ is membership grade function, $\sigma$ is standard deviation, and $a$ is optimal illumination.

The fuzzy membership grade values in Table 2 defined by experiments were incorporated into function then the unknown number in formula (4.1) can be solved as optimal luminosity value. The specific equation was shown in the following formula:

$$
\begin{aligned}
& e^{-((30-a) / \sigma)^{2}}=0.1 \\
& e^{-((20-a) / \sigma)^{2}}=0.2 .
\end{aligned}
$$

The calculation results were $a=9$ and $\sigma=2$. So that luminosity in middle segment was less than $9 \mathrm{Lx}$ was appropriate for actual demand of drivers.

\subsubsection{Result Validation}

To validate the reliability of result drivers' fuzzy evaluation experiment was performed. 23 drivers were randomly selected from all subjects to participate in this experiment. The luminosity was set at $10,8,6,4,2$, and $0 \mathrm{Lx}$, respectively. The results showed that when 
Table 3: Luminosity in entrance segment.

\begin{tabular}{lcccccc}
\hline $\begin{array}{l}\text { Distance away from } \\
\text { the entrance }\end{array}$ & 0 & 30 & 50 & 100 & 150 & 200 \\
\hline Illumination/Lx & 0 & 2 & 5 & 5 & 7 & 7 \\
\hline
\end{tabular}

Table 4: Assessment results of easing lighting entrance segment.

\begin{tabular}{lccccc}
\hline Comment & Too bright & Brighter & Appropriate & Darker & Too dark \\
\hline New entrance illumination & 0.00 & 0.00 & 0.90 & 0.5 & 0.5 \\
Original entrance illumination & 0.8 & 0.2 & 0.0 & 0.0 & 0.0 \\
\hline
\end{tabular}

the luminosity in the middle segment was less than 9 Lx membership grades of subjective luminosity \{appropriate\} levels given by drivers were all more than 0.95 . This indicated that the calculation result was effective.

\subsection{Luminosity in Entrance}

When the night ambient luminosity outside the tunnel was $0 \mathrm{Lx}$, drivers would inevitably suffer light adaptation at entrance if illumination devices are turned on. This destroyed ambient consistency of road. So the ambient luminosity should be gradually increased to 9Lx.

\subsubsection{Optimization of Entrance Illumination}

The light entered drivers eyes was ecological light reflected by every surface. The tunnel had been several reflection surfaces and was helpful to percept information. Due to diffuse reflection in tunnels, the light issued by illumination devices was more than a spot, but a domain. Meanwhile, all lamps in practices had been a certain power restriction. In consideration of convenience and feasibility, a simple solution was more reasonable. The result was shown in Table 3.

\subsubsection{Illumination Assessment in Entrance}

To verify whether this solution was effective, a comparison experiment of fuzzy assessment was performed. Randomly selected 21 drivers from all subjects were chosen to participate in this experiment. The experiment design was the same as to experiment on luminosity in middle segment. Domain $L_{e}=$ \{original entrance illumination, new entrance illumination $\}$. Fuzzy set of appropriate luminosity in entrance segment with easing illumination was $E=\{$ too bright, brighter, all right, darker, too dark $\}$, and recorded as $\left\{E_{1}, E_{2}, E_{3}, E_{4}, E_{5}\right\}$. The membership grade of each was obtained using a fuzzy statistical method, and was recorded as $R_{e}$. By taking all results into consideration, the fuzzy relation between the luminosity and its appropriateness in entrance segment with easing lighting was obtained, as shown in Table 4.

The experiment results showed that too high luminosity value in the original entrance segment might easily result in sudden environment change. Comparatively, the new illumination in entrance segment could meet the demand of drivers. 
Table 5: Luminosity in entrance exit segment.

\begin{tabular}{lccccccc}
\hline $\begin{array}{l}\text { Distance away from } \\
\text { the exit }\end{array}$ & 0 & 30 & 50 & 100 & 150 & 200 & 250 \\
\hline Illumination/Lx & 0 & 2 & 2 & 5 & 5 & 7 & 7 \\
\hline
\end{tabular}

Table 6: Assessment results of lighting easing in exit segment.

\begin{tabular}{lccccc}
\hline Comment & Too light & brighter & appropriate & darker & Too dark \\
\hline New exit illumination & 0.00 & 0.00 & 0.95 & 0.5 & 0.0 \\
Original exit illumination & 0.9 & 0.1 & 0.0 & 0.0 & 0.0 \\
\hline
\end{tabular}

\subsection{Luminosity in Exit Segment}

When drivers left the tunnel at night they would suffer dark adaptation. The luminosity at the exit that was higher dark adaptation problem would be more serious.

\subsubsection{Optimization of Exit Illumination}

The dark adaptation took longer time than light adaptation. On the contrary to entrance segment with lighting easing the optimal illumination in exit segment should be decreased gradually from $9 \mathrm{Lx}$ to $0 \mathrm{Lx}$. The result was shown in Table 5.

\subsubsection{Illumination Assessment in Exit}

Fuzzy assessment of exit segment with easing lighting was performed in the same way with that of entrance segment. The fuzzy relation between the luminosity and its appropriateness in exit segment with easing lighting was obtained, as shown in Table 6.

The results showed that luminosity value in the original exit illumination was too high. The new exit illumination proposed based on appropriate luminosity value in the middle segment could better meet the demand of drivers.

\section{Conclusions}

Measurements of luminosity in more than 100 tunnels in Shanxi and Guangdong Provinces in China showed that the luminosity in middle segment was more than 20 Lx and higher at both ends for all tunnels. But the drivers feel that luminosity value was set as $9 \mathrm{Lx}$ was appropriate in middle segment. The luminosity should be gradually increased from $0 \mathrm{Lx}$ in entrance segment, maintained constant in middle segment, and then reduced to $0 \mathrm{Lx}$ gradually in exit segment. According to these research results, night illumination of expressway long tunnels should be optimized. The optimization illumination could remove dark and light adaptation caused by luminosity difference when driving through tunnels at night. This illumination design could reduce accidents induced by visual disturbance and electricity power consumption. The following conclusions are obtained.

(1) The luminosity should be controlled less than 9Lx in the middle segment of expressway long tunnels at night. This illumination level was less than the level required in specification. 
(2) In the entrance segment the luminosity should be increased gradually from 0 Lx to 9 Lx.

(3) The luminosity should be decreased gradually from 9 Lx to 0 Lx in the exit segment.

In this paper, the optimization of night illumination in expressway long tunnels was obtained according to Chinese traffic condition and character of drivers. The conclusion was verified by lighting practice in Yongxing tunnel and Wohuwan tunnel. In fact the luminosity of each point in expressway long tunnel could not be exactly equal to the proposed ones for optical line entered the drivers eye was reflected from driving environment. In practice, the luminosity in middle segment controlled under $9 \mathrm{Lx}$ was the key problem. Such optimized night illumination in expressway long tunnel would not only eliminate the dark and light adaptation problem but also significantly reduce electricity power consumption.

\section{Acknowledgments}

This work was supported by the National Natural Science Foundation of China (51178054) the Fundamental Research Funds for the Central Universities (CHD2011JC031).

\section{References}

[1] JTJ 026.1-1999 Specifications for Design of Ventilation and Lighting of Highway Tunnel, People's Education Press, Beijing, China, 2000.

[2] W. H. Wang, W. Zhang, H. Bubb, and K. Ikeuchi, "A safety-based behavioural appraching model with various driving characteristics," Transportation Research Part C-Emerging Technologies, vol. 19, no. 6, pp. 1202-1214, 2011.

[3] H. W. Guo, W. H. Wang, W. W. Guo, X. B. Jiang, and H. Bubb, “Reliability analysis of pedestrian safety crossing in urban traffic environment," Safety Science, vol. 50, no. 4, pp. 968-973, 2012.

[4] "Norwegian Public Road Administration Road Tunnels," Norwegian, NPRA Printing Center, 2004.

[5] CIE 88:2004, Guide for the Lighting of Road Tunnels and Underpasses, International Commission on Illumination, Vienna, Austria, 2004.

[6] D. A. Schreuder and L. Swart, "Energy Saving in Tunnel Entrance Lighting," 1993, http://wenku .baidu.com/view / 46cf968ccc22bcd126ff0c2d.html.

[7] Piarc, Reduction of Operational Cost of Road Tunnels, Piarc, 1999.

[8] Y. X. Xia, "Problems and countermeasures of highway tunnel lighting," Highway Tunnel, no. 3, pp. 55-57, 2009.

[9] F. Qiu, X. J. Ma, N. T. Liu, W. Lin, and X. Z. Liu, “Disscussion on the dynamic requiring luminance in the transition section of the tunnel lighting," Zhaoming Gongcheng Xuebao, vol. 21, no. 6, pp. 13-18, 2010.

[10] G. Tu and J. Z. Chen, "Study on illumination parameter of short tunnels," Journal of Highway and Transportation Research and Development, no. 6, pp. 125-128, 2009.

[11] Y. L. Zhang, Research on illumination of medium and short tunnels in expressway [M.S. thesis], Hunan University, Changsha, China, 2008.

[12] Y. Wang, Z. Y. Guo, and Z. G. Liao, "Safety analysis for illumination design at tunnel entrance and exit," in Proceedings of the International Conference on Intelligent Computation Technology and Automation (ICICTA '10), vol. 3, pp. 255-259, May 2010.

[13] D. A. Schreuder, "Predetermination of the luminance in tunnel entrances at day," Tech. Rep., Institute for Road Safety Research SWOV, Leidschendam-Voorburg, The Netherlands, 1988.

[14] W. H. Zhao and H. X. Liu, "Drivers'visual feature variation in long-tunnel exit of expressway," in Proceeding of the First International Conference on Transportation (ICTIS '11), pp. 42-52, June 2011.

[15] R. Fukuda, "Experimental considerations on the definition of eye fixation points," Journal of Japan Ergonomics Society, vol. 28, no. 2, p. 197, 1996.

[16] H. Wang, H. X. Liu, W. H. Zhao et al., "Analysis on traffic accident feature in expressway tunnel," Highway, no. 11, pp. 144-147, 2009. 
[17] Z. G. Du, X. D. Pan, and X. B. Guo, "Experimental studies of visual adaptation during freeway tunnel's entrance and exit," Journal of Harbin Institute of Technology, vol. 39, no. 12, pp. 1998-2001, 2007.

[18] Z. G. Du, X. D. Pan, and X. B. Guo, "Evaluation index's applicatiom studies on safety at highway tunnel's entrance and exit," Journal of Tongji Institute of Technology, vol. 36, no. 3, pp. 325-329, 2008.

[19] D. A. Schreuder, "Visual performance and road safety," Tech. Rep., Institute for Road Safety Research SWOV, Leidschendam-Voorburg, The Netherlands, 1988.

[20] Z. Y. Guo, L. Q. Kong, and Z. Yang, "Investigation report of operating safety research," Tech. Rep., Institute for Road Safety Research SWOV, Leidschendam-Voorburg, The Netherlands, 1988.

[21] C. Y. Yang, Y. K. Hu, and Z. L. Chen, "Study on road lighting energy saving based on mesopic theory," China Illuminating Engineering Journal, vol. 19, no. 4, pp. 44-47, 2008. 


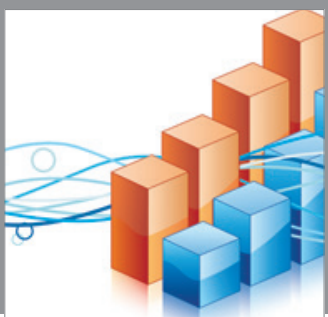

Advances in

Operations Research

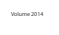

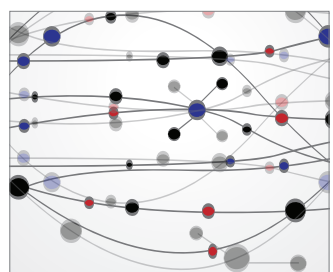

\section{The Scientific} World Journal
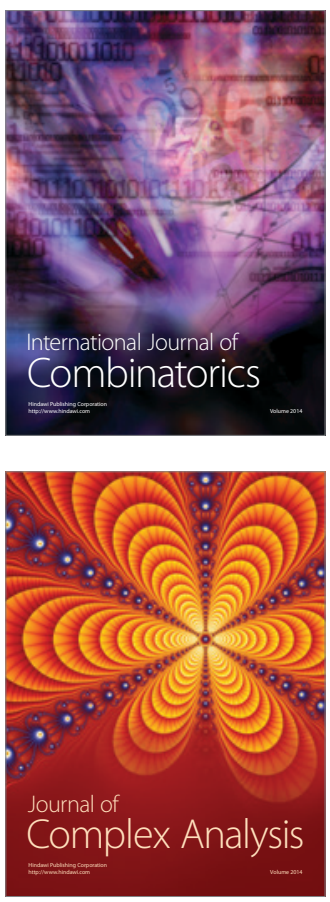

International Journal of

Mathematics and

Mathematical

Sciences
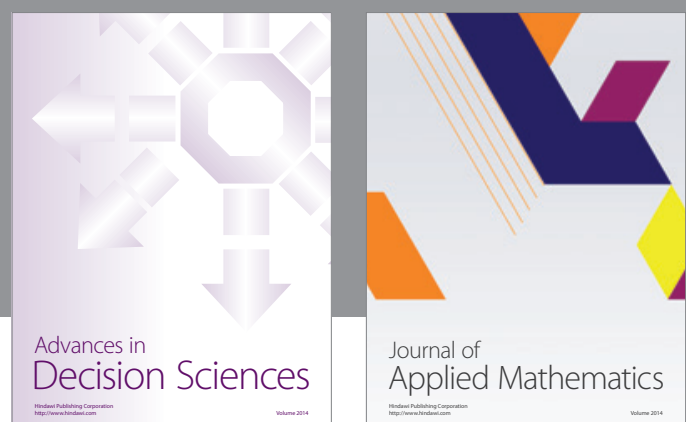

Journal of

Applied Mathematics
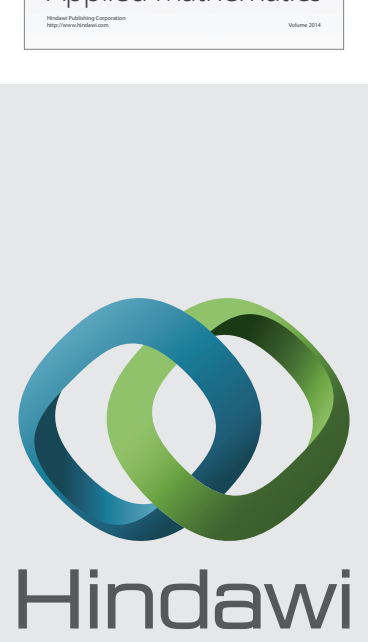

Submit your manuscripts at http://www.hindawi.com
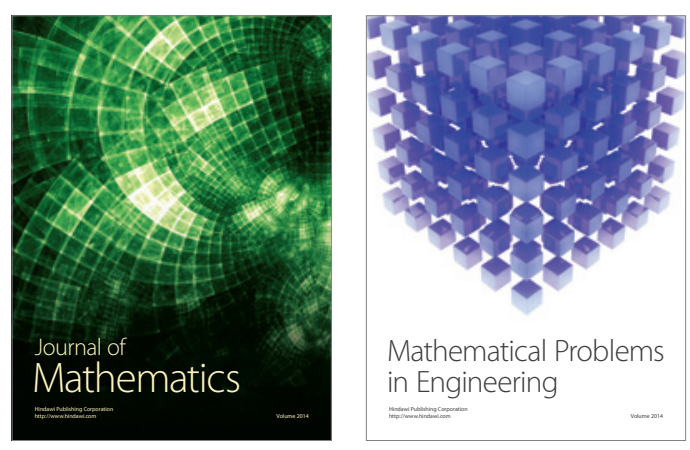

Mathematical Problems in Engineering
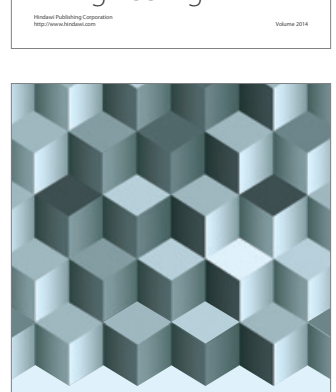

Journal of

Function Spaces
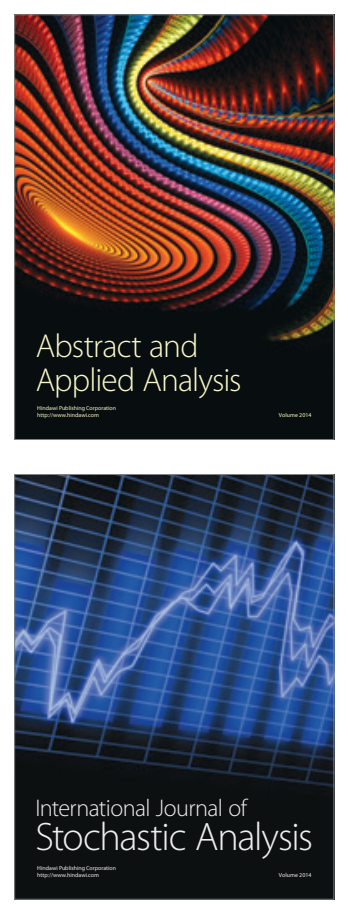

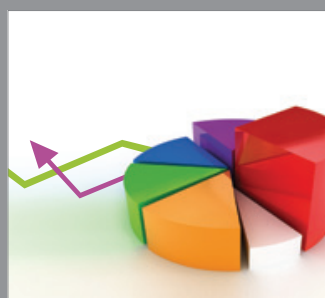

ournal of

Probability and Statistics

Promensencen
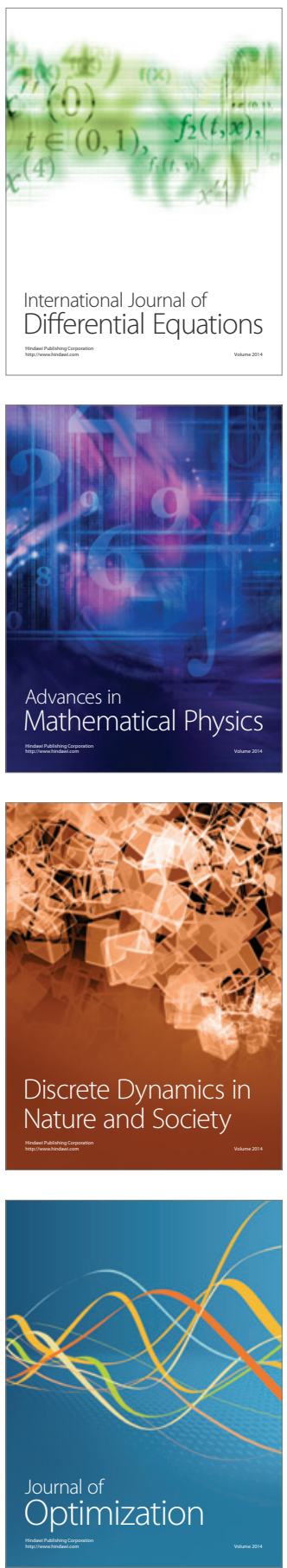